\title{
Near-colorings: non-colorable graphs and NP-completeness
}

\author{
M. Montassier and P. Ochem* \\ LIRMM (Université de Montpellier, CNRS) \\ Montpellier, France \\ montassier, ochem@lirmm.fr
}

Submitted: Jun 26, 2013; Accepted: Feb 17, 2015; Published: Mar 6, 2015

Mathematics Subject Classification: 05C15

\begin{abstract}
A graph $G$ is $\left(d_{1}, \ldots, d_{l}\right)$-colorable if the vertex set of $G$ can be partitioned into subsets $V_{1}, \ldots, V_{l}$ such that the graph $G\left[V_{i}\right]$ induced by the vertices of $V_{i}$ has maximum degree at most $d_{i}$ for all $1 \leqslant i \leqslant l$. In this paper, we focus on complexity aspects of such colorings when $l=2,3$. More precisely, we prove that, for any fixed integers $k, j, g$ with $(k, j) \neq(0,0)$ and $g \geqslant 3$, either every planar graph with girth at least $g$ is $(k, j)$-colorable or it is NP-complete to determine whether a planar graph with girth at least $g$ is $(k, j)$-colorable. Also, for every fixed integer $k$, it is NP-complete to determine whether a planar graph that is either $(0,0,0)$-colorable or non- $(k, k, 1)$-colorable is $(0,0,0)$-colorable. Additionally, we exhibit non- $(3,1)$ colorable planar graphs with girth 5 and non- $(2,0)$-colorable planar graphs with girth 7 .
\end{abstract}

\section{Introduction}

A graph $G$ is $\left(d_{1}, \ldots, d_{k}\right)$-colorable if the vertex set of $G$ can be partitioned into subsets $V_{1}, \ldots, V_{k}$ such that the graph $G\left[V_{i}\right]$ induced by the vertices of $V_{i}$ has maximum degree at most $d_{i}$ for all $1 \leqslant i \leqslant k$. This notion generalizes those of proper $k$-coloring (when $d_{1}=\cdots=d_{k}=0$ ) and $d$-improper $k$-coloring (when $d_{1}=\cdots=d_{k}=d \geqslant 1$ ).

Planar graphs are known to be $(0,0,0,0)$-colorable (Appel and Haken [1, 2]) and $(2,2,2)$-colorable (Cowen, Cowen, and Woodall [13]). The $(2,2,2)$-colorability is optimal (for any integer $k$, there exist non- $(k, k, 1)$-colorable planar graphs) and holds in the choosability case (Eaton and Hull [15] or Škrekovski [23]). Improper colorings have then been considered for planar graphs with large girth or graphs with low maximum average degree. We recall that the girth of a graph $G$, denoted by $g(G)$, is the length of a shortest cycle in

*This work was partially supported by the ANR grant EGOS 12-JS02-002-01. 
$G$, and the maximum average degree of a graph $G$, denoted by $\operatorname{mad}(\mathrm{G})$, is the maximum of the average degrees of all subgraphs of $G$, i.e. $\operatorname{mad}(G)=\max \{2|E(H)| /|V(H)|, H \subseteq G\}$.

\section{$(1,0)$-coloring.}

Glebov and Zambalaeva [20] proved that every planar graph with girth at least 16 is $(1,0)$-colorable. This was then strengthened by Borodin and Ivanova [3] who proved that every graph $G$ with $\operatorname{mad}(G)<\frac{7}{3}$ is $(1,0)$-colorable. This implies that every planar graph $G$ with girth at least 14 is $(1,0)$-colorable. Borodin and Kostochka [7] then proved that every graph $G$ with $\operatorname{mad}(G) \leqslant \frac{12}{5}$ is $(1,0)$-colorable. In particular, it follows that every planar graph with girth at least 12 is $(1,0)$-colorable. On the other hand, they constructed graphs $G$ with $\operatorname{mad}(G)$ arbitrarily close (from above) to $\frac{12}{5}$ that are not $(1,0)$-colorable; hence their upper bound on the maximum average degree is best possible. The last result was strengthened for triangle-free graphs: Kim, Kostochka, and Zhu [22] proved that triangle-free graphs $G$ satisfying $11|V(G)|-9|E(G)| \geqslant-4$ are (1,0)-colorable. This implies that planar graphs with girth at least 11 are $(1,0)$-colorable. On the other hand, Esperet, Montassier, Ochem, and Pinlou [16] proved that determining whether a planar graph with girth 9 is $(1,0)$-colorable is NP-complete. To our knowledge, the question whether all planar graphs with girth at least 10 are $(1,0)$-colorable is still open.

\section{$(k, 0)$-coloring with $k \geqslant 2$.}

Borodin, Ivanova, Montassier, Ochem, and Raspaud [4] proved that every graph $G$ with $\operatorname{mad}(G)<\frac{3 k+4}{k+2}$ is $(k, 0)$-colorable. The proof in [4] extends the one in [3] but does not work for $k=1$. Moreover, they exhibited a non- $(k, 0)$-colorable planar graph with girth 6. Finally, Borodin and Kostochka [8] proved that for $k \geqslant 2$, every graph $G$ with $\operatorname{mad}(G) \leqslant \frac{3 k+2}{k+1}$ is $(k, 0)$-colorable. This result is tight in terms of maximum average degree.

\section{$(k, 1)$-coloring.}

Recently, Borodin, Kostochka, and Yancey [9] proved that every graph with $\operatorname{mad}(G) \leqslant \frac{14}{5}$ is $(1,1)$-colorable, and the restriction on $\operatorname{mad}(G)$ is sharp. In [5], it is proven that every graph $G$ with $\operatorname{mad}(G)<\frac{10 k+22}{3 k+9}$ is $(k, 1)$-colorable for $k \geqslant 2$.

\section{$(k, j)$-coloring.}

A first step was made by Havet and Sereni [21] who showed that, for every $k \geqslant 0$, every graph $G$ with $\operatorname{mad}(G)<\frac{4 k+4}{k+2}$ is $(k, k)$-colorable (in fact $(k, k)$-choosable). More generally, they studied $k$-improper $l$-choosability and proved that every graph $G$ with $\operatorname{mad}(\mathrm{G})<l+\frac{l k}{l+k}(l \geqslant 2, k \geqslant 0)$ is $k$-improper $l$-choosable; this implies that such graphs are $(k, \ldots, k)$-colorable (where the number of partite sets is $l$ ). Borodin, Ivanova, Montassier, and Raspaud [6] gave some sufficient conditions of $(k, j)$-colorability depending on the density of the graphs using linear programming. Finally, Borodin and Kostochka [8] solved the problem for a wide range of $j$ and $k$ : let $j \geqslant 0$ and $k \geqslant 2 j+2$; every graph $G$ with $\operatorname{mad}(G) \leqslant 2\left(2-\frac{k+2}{(j+2)(k+1)}\right)$ is $(k, j)$-colorable. This result is tight in terms of the maximum average degree and improves some results in $[4,5,6]$. 
Using the fact that every planar graph $G$ with girth $g(G)$ has $\operatorname{mad}(G)<2 g(G) /(g(G)-$ $2)$, the previous results give results for planar graphs. They are summarized in Table 1 , which also shows the recent results that planar graphs with girth 5 are $(5,3)$-colorable (Choi and Raspaud [12]) and (10,1)-colorable (Choi, Choi, Jeong, and Suh [11]).

\begin{tabular}{|c|c|c|c|c|c|}
\hline girth & $(k, 0)$ & $(k, 1)$ & $(k, 2)$ & $(k, 3)$ & $(k, 4)$ \\
\hline 3,4 & $\times$ & $\times$ & $\times$ & $\times$ & $\times$ \\
\hline 5 & $\times$ & $(10,1)[11]$ & $(6,2)[8]$ & $(5,3)[12]$ & $(4,4)[21]$ \\
\hline 6 & $\times[4]$ & $(4,1)[8]$ & $(2,2)[21]$ & & \\
\hline 7 & $(4,0)[8]$ & $(1,1)[9]$ & & & \\
\hline 8 & $(2,0)[8]$ & & & & \\
\hline 11 & $(1,0)[22]$ & & & & \\
\hline
\end{tabular}

Table 1: The girth and the $(k, j)$-colorability of planar graphs. The symbol " $\times$ " means that there exist non- $(k, j)$-colorable planar graphs for every $k$.

From the previous discussion, the following questions are natural:

\section{Question 1.}

1. Are planar graphs with girth $10(1,0)$-colorable?

2. Are planar graphs with girth $7(3,0)$-colorable?

3. Are planar graphs with girth $6(1,1)$-colorable?

4. Are planar graphs with girth $5(4,1)$-colorable?

5. Are planar graphs with girth $5(2,2)$-colorable?

$\left(d_{1}, \ldots, d_{k}\right)$-coloring.

Finally we would like to mention two studies. Chang, Havet, Montassier, and Raspaud [10] gave some approximation results to Steinberg's Conjecture using $(k, j, i)$-colorings. Dorbec, Kaiser, Montassier, and Raspaud [14] studied the particular case of $\left(d_{1}, \ldots, d_{k}\right)$ coloring where the value of each $d_{i}(1 \leqslant i \leqslant k)$ is either 0 or some value $d$, making the link between $(d, 0)$-coloring $[8]$ and $(d, \ldots, d)$-coloring [21].

The aim of this paper is to provide complexity results on the subject and to obtain non-colorable planar graphs showing that some above-mentioned results are optimal.

Claim 2. There exist 2-degenerate planar graphs that are:

1. non- $(k, k)$-colorable with girth 4 , for every $k \geqslant 0$,

2. non- $(3,1)$-colorable with girth 5 ,

3. non- $(k, 0)$-colorable with girth 6 , 
4. non-(2,0)-colorable with girth $\%$.

Claim 2.4 shows that the $(2,0)$-colorability of planar graphs with girth at least 8 [8] is a tight result. Claim 2.3 has been obtained in [4] and the corresponding graph is depicted in Figure 1.

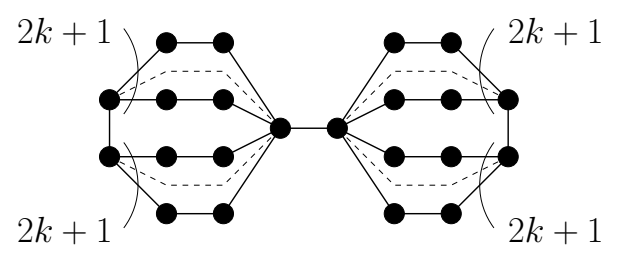

Figure 1: A non- $(k, 0)$-colorable planar graph with girth 6 [4].

Theorem 3. Let $k, j$, and $g$ be fixed integers such that $(k, j) \neq(0,0)$ and $g \geqslant 3$. Either every planar graph with girth at least $g$ is $(k, j)$-colorable or it is NP-complete to determine whether a planar graph with girth at least $g$ is $(k, j)$-colorable.

Theorem 4. Let $k$ be a fixed integer. It is NP-complete to determine whether a 3degenerate planar graph that is either $(0,0,0)$-colorable or non- $(k, k, 1)$-colorable is $(0,0,0)$ colorable.

We construct a non- $(k, k)$-colorable planar graph with girth 4 in Section 2 , a non$(3,1)$-colorable planar graph with girth 5 in Section 3 , and a non- $(2,0)$-colorable planar graph with girth 7 in Section 4 . We prove Theorem 3 in Section 5 and we prove Theorem 4 in Section 6.

\section{Notation.}

In the following, when we consider a $\left(d_{1}, \ldots, d_{k}\right)$-coloring of a graph $G$, we color the vertices of $V_{i}$ with color $d_{i}$ for $1 \leqslant i \leqslant k$ : for example in a $(3,0)$-coloring, we will use color 3 to color the vertices of $V_{1}$ inducing a subgraph with maximum degree 3 and use color 0 to color the vertices of $V_{2}$ inducing a stable set. A vertex is said to be colored $i^{j}$ if it is colored $i$ and has $j$ neighbors colored $i$, that is, it has degree $j$ in the subgraph induced by its color. A vertex is saturated if it is colored $i^{i}$, that is, if it has maximum degree in the subgraph induced by its color. A cycle (resp. face) of length $k$ is called a $k$-cycle (resp. $k$-face). A $k$-vertex (resp. $k^{-}$-vertex, $k^{+}$-vertex) is a vertex of degree $k$ (resp. at most $k$, at least $k$ ). The minimum degree of a graph $G$ is denoted by $\delta(G)$.

\section{A non- $(k, k)$-colorable planar graph with girth 4}

For every $k \geqslant 0$, we construct a non- $(k, k)$-colorable planar graph $J_{4}$ with girth 4 . Let $H_{x, y}$ be a copy of $K_{2,2 k+1}$, as depicted in Figure 2. In any $(k, k)$-coloring of $H_{x, y}$, the 
vertices $x$ and $y$ must receive the same color. We obtain $J_{4}$ from a vertex $u$ and a star $S$ with center $v_{0}$ and $k+1$ leaves $v_{1}, \ldots, v_{k+1}$ by linking $u$ to every vertex $v_{i}$ with a copy $H_{u, v_{i}}$ of $H_{x, y}$. The graph $J_{4}$ is not $(k, k)$-colorable: by the property of $H_{x, y}$, every vertex $v_{i}$ should get the same color as $u$. This gives a monochromatic $S$, which is forbidden. Notice that $J_{4}$ is a planar graph with girth 4 and is 2-degenerate.
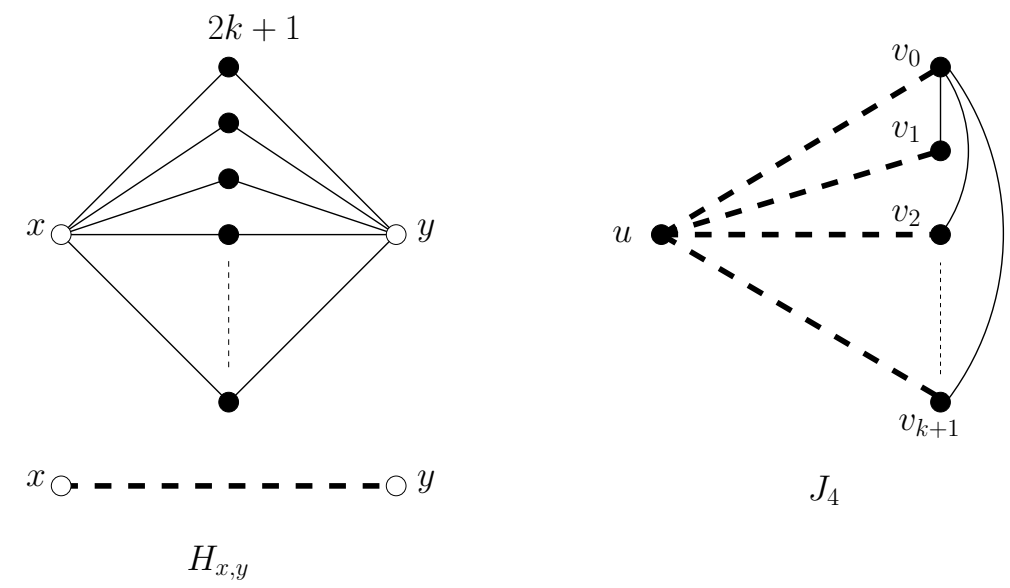

Figure 2: A non- $(k, k)$-colorable planar graph with girth 4 .

\section{A non-(3, 1)-colorable planar graph with girth 5}

We construct a non- $(3,1)$-colorable planar graph $J_{5}$ with girth 5 . Consider the graph $H_{x, y}$ depicted in Figure 3. If $x$ and $y$ are colored 3 but have no neighbor colored 3, then it is
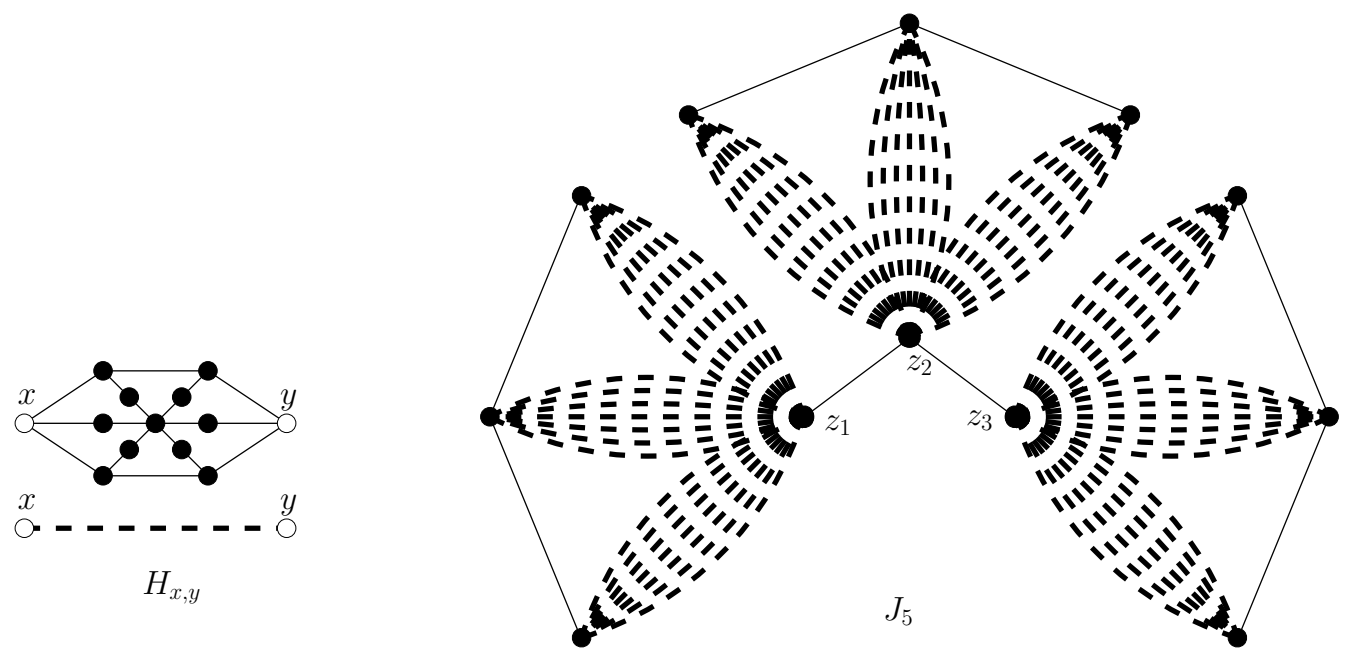

Figure 3: A non-(3,1)-colorable planar graph with girth 5 . 
not possible to extend this partial coloring to $H_{x, y}$. Now, we construct the graph $S_{z}$ as follows. Let $z$ be a vertex and $t_{1} t_{2} t_{3}$ be a path on three vertices. Take 21 copies $H_{x_{i}, y_{j}}$ of $H_{x, y}$ with $1 \leqslant i \leqslant 7$ and $1 \leqslant j \leqslant 3$. Identify every $x_{i}$ with $z$ and identify every $y_{i}$ with $t_{i}$. Finally, we obtain $J_{5}$ from three copies $S_{z_{1}}, S_{z_{2}}$, and $S_{z_{3}}$ of $S_{z}$ by adding the edges $z_{1} z_{2}$ and $z_{2} z_{3}$ (Figure 3). Notice that $J_{5}$ is planar with girth 5 and is 2-degenerate. Let us show that $J_{5}$ is not $(3,1)$-colorable. In every $(3,1)$-coloring of $J_{5}$, the path $z_{1} z_{2} z_{3}$ contains a vertex $z$ colored 3 . In the copy of $S_{z}$ corresponding to $z$, the path $t_{1} t_{2} t_{3}$ contains a vertex $t$ colored 3 . Since $z$ (resp. $t$ ) has at most 3 neighbors colored 3 , one of the seven copies of $H_{x, y}$ between $z$ and $t$, does not contain a neighbor of $z$ or $t$ colored 3 . This copy of $H_{x, y}$ is not $(3,1)$-colorable, and thus $J_{5}$ is not $(3,1)$-colorable.

\section{A non-(2,0)-colorable planar graph with girth 7}

We construct of a non- $(2,0)$-colorable planar graph $J_{7}$ with girth 7 . Consider the graphs $T_{x, y, z}$ and $S$ in Figure 4.
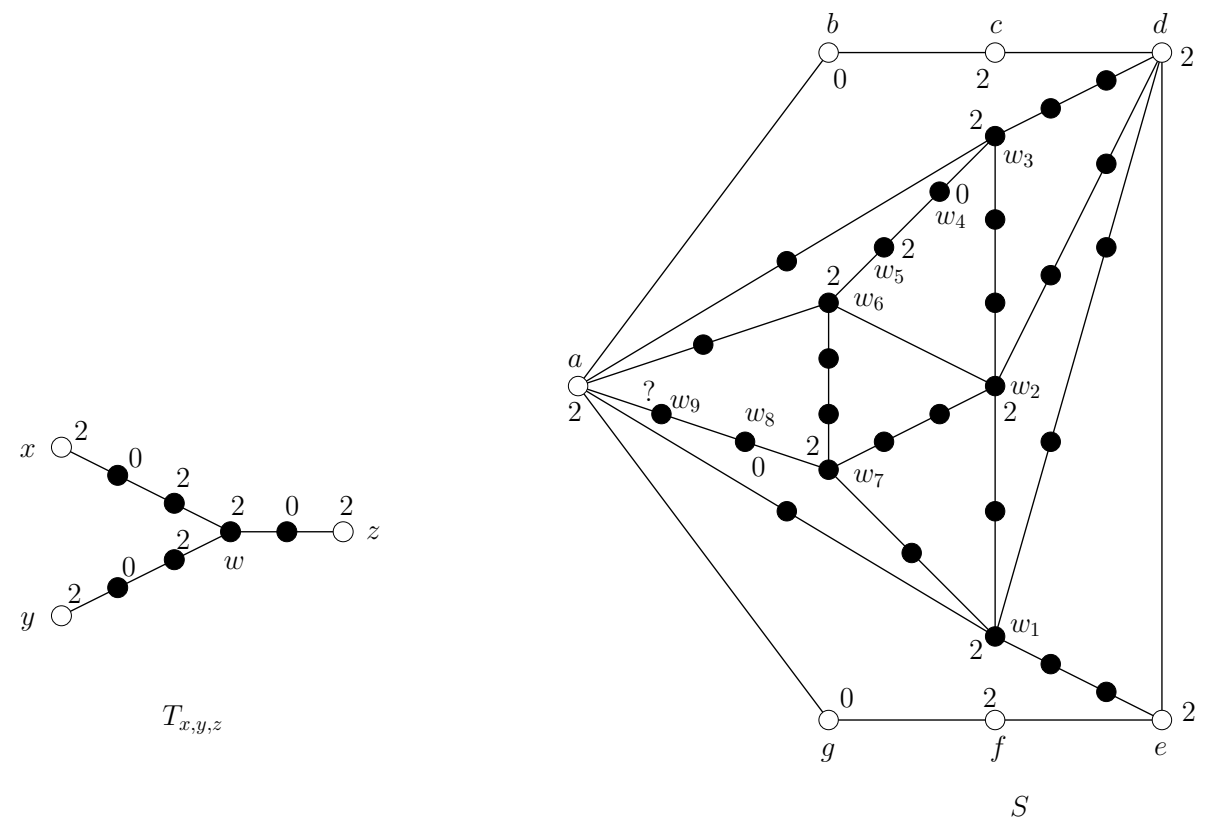

Figure 4: The graphs $T_{x, y, z}$ and $S$.

If the vertices $x, y$, and $z$ of $T_{x, y, z}$ are colored 2 and have no neighbor colored 2 , then $w$ is colored $2^{2}$. Suppose that the vertices $a, b, c, d, e, f, g$ of $S$ are respectively colored 2 , $0,2,2,2,2,0$, and that $a$ has no neighbor colored 2 . Using successively the property of $T_{x, y, z}$, we have that $w_{1}, w_{2}$, and $w_{3}$ must be colored $2^{2}$. It follows that $w_{4}$ is colored $0, w_{5}$ is colored 2, and so $w_{6}$ is colored $2^{2}$. Again, by the property of $T_{x, y, z}, w_{7}$ must be colored $2^{2}$. Finally, $w_{8}$ must be colored 0 and there is no choice of color for $w_{9}$. Hence, such a coloring of the outer 7-cycle abcdef $g$ cannot be extended. 
The graph $H_{z}$ depicted on the left of Figure 5 is obtained as follows.
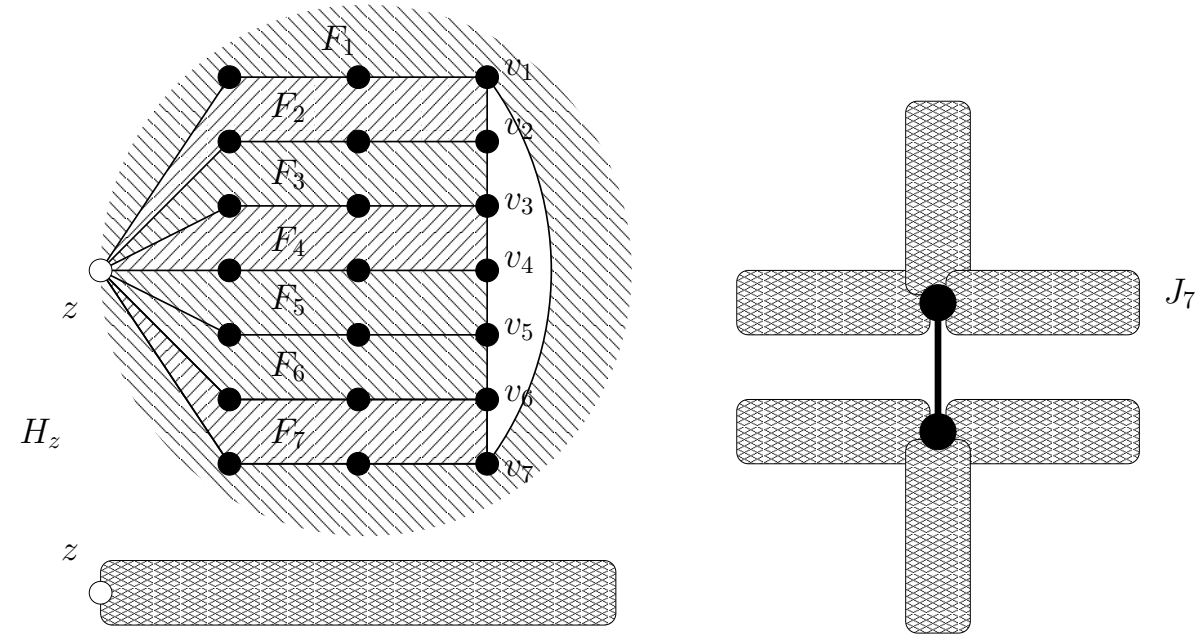

Figure 5: The graphs $H_{z}$ and $J_{7}$.

We link a vertex $z$ to every vertex of a 7 -cycle $v_{1} \ldots v_{7}$ with a path of three edges. Then we embed the graph $S$ in every 7 -face $F_{i}(1 \leqslant i \leqslant 7)$ incident to $z$ by identifying the outer 7-cycle of $S$ with the 7-cycle of $F_{i}$ such that $a$ is identified to $z$. Finally, the graph $J_{7}$ depicted on the right of Figure 5 is obtained from two adjacent vertices $u$ and $v$ and six copies $H_{z_{1}}, \ldots, H_{z_{6}}$ of $H_{z}$ by identifying $z_{1}, z_{2}, z_{3}$ with $u$ and $z_{4}, z_{5}, z_{6}$ with $v$. Notice that $J_{7}$ is planar with has girth 7 . Let us prove that $J_{7}$ is not $(2,0)$-colorable.

- We assume that $u$ is colored 2 since $u$ and $v$ cannot be both colored 0 .

- In one of the three copies of $H_{z}$ attached to $u$, say $H_{z_{1}}, u$ has no neighbor colored 2 .

- Since a 7-cycle is not 2-colorable, the 7-cycle $v_{1} \ldots v_{7}$ of $H_{z_{1}}$ contains a monochromatic edge colored 2 , say $v_{1} v_{2}$.

- The coloring of the face $F_{2}$ cannot be extended to the copy of $S$ embedded in $F_{2}$.

\section{$5 \quad$ NP-completeness of $(k, j)$-colorings}

Let $g_{k, j}$ be the largest integer $g$ such that there exists a planar graph with girth $g$ that is not $(k, j)$-colorable. Because of large odd cycles, $g_{0,0}$ is not defined. For $(k, j) \neq(0,0)$, we have $4 \leqslant g_{k, j} \leqslant 10$ by the example in Figure 2 and the result that planar graphs with girth at least 11 are $(0,1)$-colorable [22]. We prove this equivalent form of Theorem 3 :

Theorem 5. Let $k$ and $j$ be fixed integers such that $(k, j) \neq(0,0)$. It is NP-complete to determine whether a planar graph with girth $g_{k, j}$ is $(k, j)$-colorable. 
Let us define the partial order $\preceq$. Let $n_{3}(G)$ be the number of $3^{+}$-vertices in $G$. For any two graphs $G_{1}$ and $G_{2}$, we have $G_{1} \prec G_{2}$ if and only if at least one of the following conditions holds:

- $\left|V\left(G_{1}\right)\right|<\left|V\left(G_{2}\right)\right|$ and $n_{3}\left(G_{1}\right) \leqslant n_{3}\left(G_{2}\right)$.

- $n_{3}\left(G_{1}\right)<n_{3}\left(G_{2}\right)$.

Note that the partial order $\preceq$ is well-defined and is a partial linear extension of the subgraph poset. The following lemma is useful.

Lemma 6. Let $k$ and $j$ be fixed integers such that $(k, j) \neq(0,0)$. There exists a planar graph $G_{k, j}$ with girth $g_{k, j}$, minimally non- $(k, j)$-colorable for the subgraph order, such that $\delta\left(G_{k, j}\right)=2$.

Proof. We have $\delta\left(G_{k, j}\right) \geqslant 2$, since a non- $(k, j)$-colorable graph that is minimal for the subgraph order does not contain a $1^{-}$-vertex. Supppose that for some pair $(k, j)$, we construct a 2-degenerate non- $(k, j)$-colorable planar graph with girth $g_{k, j}$. Then this graph contains a (not necessarily proper) minimally non- $(k, j)$-colorable subgraph with minimum degree 2 . Thus, we can prove the lemma for the following pairs $(k, j)$ by using Claim 2.

- Pairs $(k, j)$ such that $g_{k, j} \leqslant 4$ : We actually have $g_{k, j}=4$ by Claim 2.1 .

- Pairs $(k, j)$ such that $g_{k, j} \geqslant 6$ : Indeed, a planar graph with girth at least 6 is 2degenerate. In particular, Claim 2.3 shows that $g_{k, 0} \geqslant 6$, so the lemma is proved for all pairs $(k, 0)$.

- Pairs $(k, 1)$ such that $1 \leqslant k \leqslant 3$ : If $g_{k, j} \geqslant 6$, then we are in a previous case. Otherwise, we have $g_{k, j}=5$ by Claim 2.2.

The remaining pairs satisfy $g_{k, j}=5$. There are two types of remaining pairs $(k, j)$ :

- Type 1: $k \geqslant 4$ and $j=1$.

- Type $2: 2 \leqslant j \leqslant k$.

Consider a planar graph $G$ with girth 5 that is non- $(k, j)$-colorable and is minimal for the order $\preceq$. Suppose for contradiction that $G$ does not contain a 2 -vertex. Also, suppose that $G$ contains a 3 -vertex $a$ adjacent to three $4^{-}$-vertices $a_{1}, a_{2}$, and $a_{3}$. For colorings of type 1, we can extend to $G$ a coloring of $G \backslash\{a\}$ by assigning to $a$ the color of improperty at least 4. For colorings of type 2, we consider the graph $G^{\prime}$ obtained from $G \backslash\{a\}$ by adding three 2 -vertices $b_{1}, b_{2}$, and $b_{3}$ adjacent to, respectively, $a_{2}$ and $a_{3}, a_{1}$ and $a_{3}, a_{1}$ and $a_{2}$. Notice that $G^{\prime} \preceq G$, so $G^{\prime}$ admits a coloring $c$ of type 2 . We can extend to $G$ the coloring of $G \backslash\{a\}$ induced by $c$ as follows. If $a_{1}, a_{2}$, and $a_{3}$ have the same color, then we assign to $a$ the other color. Otherwise, we assign to $a$ the color that appears at least twice among the colors of $b_{1}, b_{2}$, and $b_{3}$. Now, since $G$ does not contain a 2 -vertex nor a 
3 -vertex adjacent to three $4^{-}$-vertices, we have $\operatorname{mad}(\mathrm{G}) \geqslant \frac{10}{3}$. This can be seen using the discharging procedure such that the initial charge of each vertex is its degree and every $5^{+}$-vertex gives $\frac{1}{3}$ to each adjacent 3 -vertex. The final charge of a 3 -vertex is at least $3+\frac{1}{3}=\frac{10}{3}$, the final charge of a 4 -vertex is $4>\frac{10}{3}$, and the final charge of a $k$-vertex with $k \geqslant 5$ is at least $k-k \times \frac{1}{3}=\frac{2 k}{3} \geqslant \frac{10}{3}$. Now, $\operatorname{mad}(\mathrm{G}) \geqslant \frac{10}{3}$ contradicts the fact that $G$ is a planar graph with girth 5 , and this contradiction shows that $G$ contains a 2-vertex.

We are ready to prove Theorem 5 . The case of $(1,0)$-coloring is proved in a stronger form which takes into account restrictions on both the girth and the maximum degree of the input planar graph [16].

Proof of the case $(k, 0), k \geqslant 2$.

We consider a graph $G_{k, 0}$ as described in Lemma 6 , which contains a path $u x v$ where $x$ is a 2-vertex. By minimality, any $(k, 0)$-coloring of $G_{k, 0} \backslash\{x\}$ is such that $u$ and $v$ get distinct saturated colors. Let $G$ be the graph obtained from $G_{k, 0} \backslash\{x\}$ by adding three 2-vertices $x_{1}, x_{2}$, and $x_{3}$ to create the path $u x_{1} x_{2} x_{3} v$. So any $(k, 0)$-coloring of $G$ is such that $x_{2}$ is colored $k^{1}$. To prove the NP-completeness, we reduce the $(k, 0)$-coloring problem to the $(1,0)$-coloring problem. Let $I$ be a planar graph with girth $g_{1,0}$. For every vertex $s$ of $I$, add $(k-1)$ copies of $G$ such that the vertex $x_{2}$ of each copy is adjacent to $s$, to obtain the graph $I^{\prime}$. By construction, $I^{\prime}$ is $(k, 0)$-colorable if and only if $I$ is $(1,0)$-colorable. Moreover, $I^{\prime}$ is planar, and since $g_{k, 0} \leqslant g_{1,0}$, the girth of $I^{\prime}$ is $g_{k, 0}$.

Proof of the case $(1,1)$.

By Claim 2.2 and $[9], g_{1,1}$ is either 5 or 6 . There exist two independent proofs $[17,19]$ that $(1,1)$-coloring is NP-complete for triangle-free planar graphs with maximum degree 4 . We use a reduction from that problem to prove that $(1,1)$-coloring is NP-complete for planar graphs with girth $g_{1,1}$. We consider a graph $G_{1,1}$ as described in Lemma 6, which contains a path $u x v$ where $x$ is a 2-vertex. By minimality, any $(1,1)$-coloring of $G_{1,1} \backslash\{x\}$ is such that $u$ and $v$ get distinct saturated colors. Let $G$ be the graph obtained from $G_{1,1} \backslash\{x\}$ by adding a vertex $u^{\prime}$ adjacent to $u$ and a vertex $v^{\prime}$ adjacent to $v$. So any $(1,1)$-coloring of $G$ is such that $u^{\prime}$ and $v^{\prime}$ get distinct colors and $u^{\prime}$ (resp. $v^{\prime}$ ) has a color distinct from the color of its (unique) neighbor. We construct the graph $E_{a, b}$ from two vertices $a$ and $b$ and two copies of $G$ such that $a$ is adjacent to the vertices $u^{\prime}$ of both copies of $G$ and $b$ is adjacent to the vertices $v^{\prime}$ of both copies of $G$. There exists a $(1,1)$-coloring of $E_{a, b}$ such that $a$ and $b$ have distinct colors and neither $a$ nor $b$ is saturated. There exists a $(1,1)$-coloring of $E_{a, b}$ such that $a$ and $b$ have the same color. Moreover, in every $(1,1)$-coloring of $E_{a, b}$ such that $a$ and $b$ have the same color, both $a$ and $b$ are saturated.

The reduction is as follows. Let $I$ be a planar graph. For every edge $(p, q)$ of $I$, replace $(p, q)$ by a copy of $E_{a, b}$ such that $a$ is identified with $p$ and $b$ is identified with $q$, to obtain the graph $I^{\prime}$. By the properties of $E_{a, b}, I$ is $(1,1)$-colorable if and only if $I^{\prime}$ is $(1,1)$-colorable. Moreover, $I^{\prime}$ is planar with girth $g_{1,1}$.

Proof of the case $(k, j)$.

We consider a graph $G_{k, j}$ as described in Lemma 6 , which contains a path $u x v$ where $x$ 
is a 2-vertex. By minimality, any $(k, j)$-coloring of $G_{k, j} \backslash\{x\}$ is such that $u$ and $v$ get distinct saturated colors. Let $G$ be the graph obtained from $G_{k, j} \backslash\{x\}$ by adding a vertex $u^{\prime}$ adjacent to $u$ and a vertex $v^{\prime}$ adjacent to $v$. So any $(k, j)$-coloring of $G$ is such that $u^{\prime}$ and $v^{\prime}$ get distinct colors and $u^{\prime}$ (resp. $v^{\prime}$ ) has a color distinct from the color of its (unique) neighbor. Let $t=\min (k-1, j)$. To prove the NP-completeness, we reduce the $(k, j)$-coloring to the $(k-t, j-t)$-coloring. Thus the case $(k, k)$ reduces to the case $(1,1)$ which is NP-complete, and the case $(k, j)$ with $j<k$ reduces to the case $(k-j, 0)$ which is NP-complete. The reduction is as follows. Let $I$ be a planar graph with girth $g_{k-t, j-t}$. For every vertex $s$ of $I$, add $t$ copies of $G$ such that the vertices $u^{\prime}$ and $v^{\prime}$ of each copy is adjacent to $s$, to obtain the graph $I^{\prime}$. By construction, $I$ is $(k-t, j-t)$-colorable if and only if $I^{\prime}$ is $(k, j)$-colorable. Moreover, $I^{\prime}$ is planar, and since $g_{k, j} \leqslant g_{k-t, j-t}$, the girth of $I^{\prime}$ is $g_{k, j}$.

\section{NP-completeness of $(k, j, i)$-colorings}

In this section, we prove Theorem 4 using a reduction from 3 -colorability, i.e. $(0,0,0)$ colorability, which is NP-complete for planar graphs [18].

Let $E$ be the graph depicted in Fig 6 . The graph $E^{\prime}$ is obtained from $2 k-1$ copies of $E$ by identifying the edge $a b$ of all copies. Take 4 copies $E_{1}^{\prime}, E_{2}^{\prime}, E_{3}^{\prime}$, and $E_{4}^{\prime}$ of $E^{\prime}$ and consider a triangle $T$ formed by the vertices $y_{0}, x_{0}, x_{1}$ in one copy of $E$ in $E_{1}^{\prime}$. The graph $E^{\prime \prime}$ is obtained by identifying the edge $y_{0} x_{0}$ (resp. $y_{0} x_{1}, x_{0} x_{1}$ ) of $T$ with the edge $a b$ of $E_{2}^{\prime}$ (resp. $E_{3}^{\prime}, E_{4}^{\prime}$ ). The edge $a b$ of $E_{1}^{\prime}$ is then said to be the edge $a b$ of $E^{\prime \prime}$.

\section{Lemma 7.}

1. $E^{\prime \prime}$ admits a $(0,0,0)$-coloring.

2. $E^{\prime}$ does not admit a $(k, k, 1)$-coloring such that $a$ and $b$ have a same color of improperty $k$.

3. $E^{\prime \prime}$ does not admit $a(k, k, 1)$-coloring such that $a$ and $b$ have the same color.

Proof.

1. The following $(0,0,0)$-coloring $c$ of $E$ is unique up to permutation of colors: $c(a)=$ $c\left(x_{i}\right)=1$ for even $i, c(b)=c\left(y_{i}\right)=2$ for even $i$, and $c\left(x_{i}\right)=c\left(y_{i}\right)=3$ for odd $i$. This coloring can be extended into a $(0,0,0)$-coloring of $E^{\prime}$ and $E^{\prime \prime}$.

2. Let $k_{1}, k_{2}$, and 1 denote the colors in a potential $(k, k, 1)$-coloring $c$ of $E^{\prime}$ such that $c(a)=c(b)=k_{1}$. By the pigeon-hole principle, one of the $2 k-1$ copies of $E$ in $E^{\prime}$, say $E^{*}$, is such that $a$ and $b$ are the only vertices with color $k_{1}$. So, one of the vertices $x_{0}, y_{0}$, and $x_{3 k+3+t}$ in $E^{*}$ must get color $k_{2}$ since they cannot all get color 1 . We thus have a vertex $v_{1} \in\{a, b\}$ colored $k_{1}$ and vertex $v_{2} \in\left\{x_{0}, y_{0}, x_{3 k+3+t}\right\}$ colored $k_{2}$ in $E^{*}$ which both dominate a path on at least $3 k+3$ vertices. This path contains no vertex colored $k_{1}$ since it is in $E^{*}$. Moreover, it contains at most $k$ vertices colored 


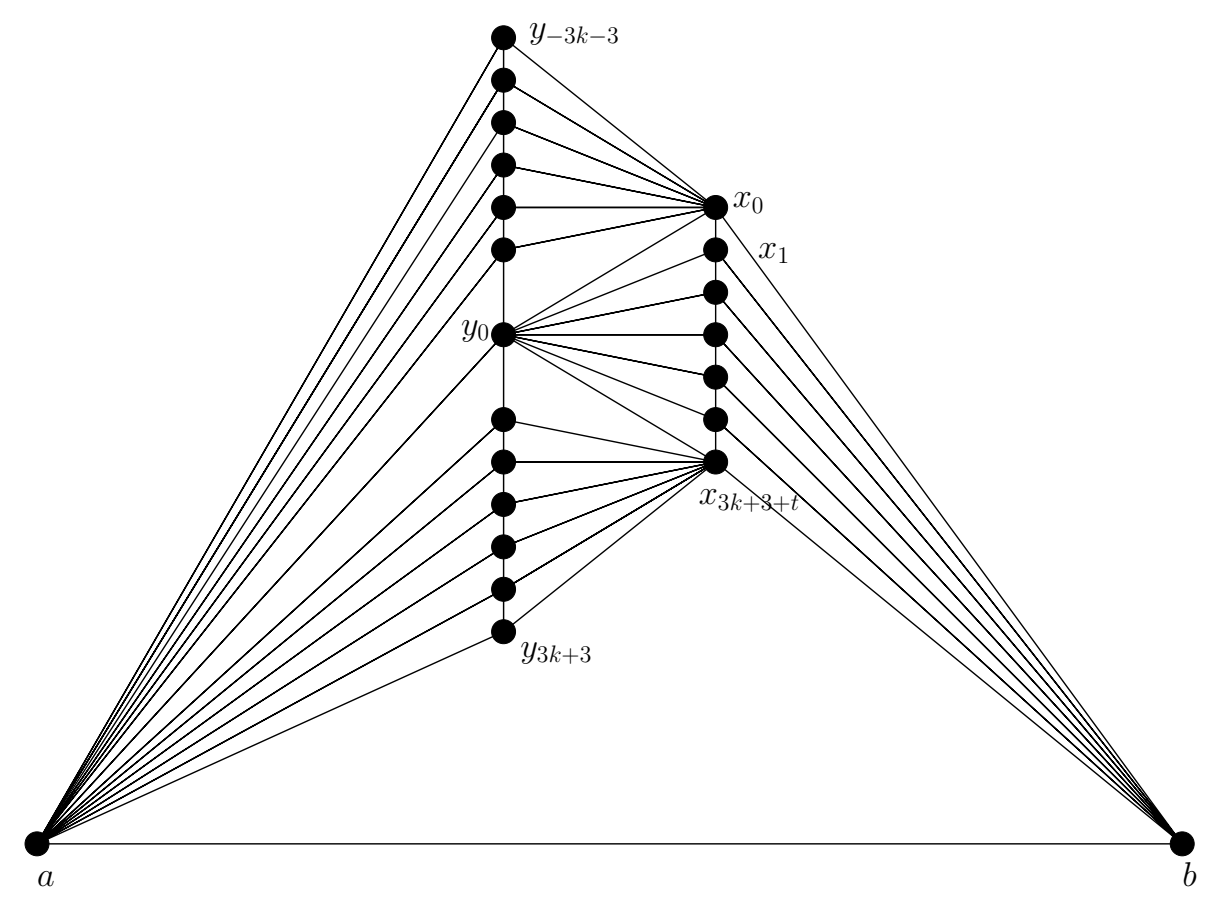

Figure 6: The graph $E$. We take $t=0$ if $k$ is odd and $t=1$ if $k$ is even, so that $3 k+3+t$ is even.

$k_{2}$. On the other hand, every set of 3 consecutive vertices in this path contains at least one vertex colored $k_{2}$, so it contains at least $\frac{3 k+3}{3}=k+1$ vertices colored $k_{2}$. This contradiction shows that $E^{\prime}$ does not admit a $(k, k, 1)$-coloring such that $a$ and $b$ have a same color of improperty $k$.

3. By the previous item and by construction of $E^{\prime \prime}$, if $E^{\prime \prime}$ admits a $(k, k, 1)$-coloring $c$ such that $c(a)=c(b)$, then $c(a)=c(b)=1$. We thus have that $\left\{c\left(y_{0}\right), c\left(x_{0}\right), c\left(x_{1}\right)\right\} \subset$ $\left\{k_{1}, k_{2}\right\}$. This implies that at least one edge of the triangle $T$ is monochromatic with a color of improperty $k$. By the previous item, the coloring $c$ cannot be extended to the copy of $E^{\prime}$ attached to that monochromatic edge. This shows that $E^{\prime \prime}$ does not admit a $(k, k, 1)$-coloring such that $a$ and $b$ have the same color.

For every fixed integer $k$, we give a polynomial construction that transforms every planar graph $G$ into a planar graph $G^{\prime}$ such that $G^{\prime}$ is $(0,0,0)$-colorable if $G$ is $(0,0,0)$ colorable and $G^{\prime}$ is not $(k, k, 1)$-colorable otherwise. The graph $G^{\prime}$ is obtained from $G$ by identifying every edge of $G$ with the edge $a b$ of a copy of $E^{\prime \prime}$. If $G$ is $(0,0,0)$-colorable, then this coloring can be extended into a $(0,0,0)$-coloring of $G^{\prime}$ by Lemma 7.1. If $G$ is not $(0,0,0)$-colorable, then every $(k, k, 1)$-coloring $G$ contains a monochromatic edge $u v$, and then the copy of $E^{\prime \prime}$ corresponding to $u v$ (and thus $G^{\prime}$ ) does not admit a $(k, k, 1$ )coloring by Lemma 7.3. The instance graph $G$ in the proof that $(0,0,0)$-coloring is NP-complete [18] is 3-degenerate. Then by construction, $G^{\prime}$ is also 3-degenerate. 


\section{References}

[1] K. Appel and W. Haken. Every planar map is four colorable. Part I. Discharging. Illinois J. Math., 21:429-490, 1977.

[2] K. Appel and W. Haken. Every planar map is four colorable. Part II. Reducibility. Illinois J. Math., 21:491-567, 1977.

[3] O.V. Borodin and A.O. Ivanova. Near proper 2-coloring the vertices of sparse graphs. Diskretn. Anal. Issled. Oper., 16(2):16-20, 2009.

[4] O.V. Borodin, A.O. Ivanova, M. Montassier, P. Ochem, and A. Raspaud. Vertex decompositions of sparse graphs into an edgeless subgraph and a subgraph of maximum degree at most $k$. J. Graph Theory, 65(2):83-93, 2010.

[5] O.V. Borodin, A.O. Ivanova, M. Montassier, and A. Raspaud. ( $k, 1)$-coloring of sparse graphs. Discrete Math., 312(6):1128-1135, 2012.

[6] O.V. Borodin, A.O. Ivanova, M. Montassier, and A. Raspaud. ( $k, j)$-coloring of sparse graphs. Discrete Appl. Math., 159(17):1947-1953, 2011.

[7] O.V. Borodin and A.V. Kostochka. Vertex partitions of sparse graphs into an independent vertex set and subgraph of maximum degree at most one. Sibirsk. Mat. Zh., 52(5):1004-1010, 2011. (in Russian.)

[8] O.V. Borodin and A.V. Kostochka. Defective 2-coloring of sparse graphs. J. Combin. Theory S. B, 104:72-80, 2014.

[9] O.V. Borodin, A.V. Kostochka, and M. Yancey. On 1-improper 2-coloring of sparse graphs. Discrete Math., 313(22):2638-2649, 2013.

[10] G.J. Chang, F. Havet, M. Montassier, and A. Raspaud. Steinberg's Conjecture and near-colorings. Research Report RR-7669, INRIA, 2011.

[11] H. Choi, I. Choi, J. Jeong, and G. Suh. $(1, k)$-coloring of graphs with girth at least 5 on a surface. arXiv: 1412.0344

[12] I. Choi and A. Raspaud. Planar graphs with girth at least 5 are $(3,5)$-colorable. Discrete Math., 318(4):661-667, 2015.

[13] L.J. Cowen, R.H. Cowen, and D.R. Woodall. Defective colorings of graphs in surfaces: partitions into subgraphs of bounded valency. J. Graph Theory, 10(2):187-195, 1986.

[14] P. Dorbec, T. Kaiser, M. Montassier, and A. Raspaud. Limits of near-coloring of sparse graphs. J. Graph Theory, 75(2):191-202, 2014.

[15] N. Eaton and T. Hull. Defective list colorings of planar graphs. Bull. Inst. Combin. Appl., 25:79-87, 1999.

[16] L. Esperet, M. Montassier, P. Ochem, and A. Pinlou. A complexity dichotomy for the coloring of sparse graphs. J. Graph Theory, 73(1):85-102, 2013.

[17] J. Fiala, K. Jansen, V.B. Le, and E. Seidel. Graph subcolorings: complexity and algorithms. SIAM J. Discrete Math., 16(4):635-650, 2003. 
[18] M.R. Garey, D.S. Johnson, and L.J. Stockmeyer, Some simplified NP-complete graph problems. Theor. Comput. Sci., 1:237-267, 1976.

[19] J. Gimbel and C. Hartman. Subcolorings and the subchromatic number of a graph. Discrete Math., 272:139-154, 2003.

[20] A.N. Glebov, D.Zh. Zambalaeva. Path partitions of planar graphs. Sib. Elektron. Mat. Izv., 4:450-459, 2007. (in Russian.) http://semr.math.nsc.ru

[21] F. Havet and J.-S. Sereni. Improper choosability of graphs and maximum average degree. J. Graph Theory, 52:181-199, 2006.

[22] J. Kim, A.V. Kostochka, and X. Zhu. Improper coloring of sparse graphs with a given girth, I: (0,1)-colorings of triangle-free graphs. European J. Combin., 42:26-48, 2014.

[23] R. Škrekovski. List improper coloring of planar graphs. Combin. Probab. Comput., 8:293-299, 1999. 